\title{
Diversity-Multiplexing-Nulling Trade-Off Analysis of Multiuser MIMO System for Intercell Interference Coordination
}

\author{
Jinwoo Kim ${ }^{1}$ and Chung G. Kang ${ }^{2}$ \\ ${ }^{1}$ IT and Mobile Communication Division, Samsung Electronics, Soowon, Republic of Korea \\ ${ }^{2}$ School of Electrical Engineering, Korea University, Seoul, Republic of Korea \\ Correspondence should be addressed to Chung G. Kang; ccgkang@korea.ac.kr
}

Received 16 September 2017; Accepted 6 November 2017; Published 10 December 2017

Academic Editor: Mostafa Zaman Chowdhury

Copyright (c) 2017 Jinwoo Kim and Chung G. Kang. This is an open access article distributed under the Creative Commons Attribution License, which permits unrestricted use, distribution, and reproduction in any medium, provided the original work is properly cited.

\begin{abstract}
A fundamental performance trade-off of multicell multiuser multiple-input multiple-output (MU-MIMO) systems is explored for achieving intercell and intracell interference-free conditions. In particular, we analyze the three-dimensional diversitymultiplexing-nulling trade-off (DMNT) among the diversity order (i.e., the slope of the error performance curve), multiplexing order (i.e., the number of users that are simultaneously served by MU-MIMO), and nulling order (i.e., the number of users with zero interference in a victim cell). This trade-off quantifies the performance of MU-MIMO in terms of its diversity and multiplexing order, while nulling the intercell interference toward the victim cell in the neighbor. First, we design a precoding matrix to mitigate both intercell and intracell interference for a linear precoding-based MU-MIMO system. Then, the trade-off relationship is obtained by analyzing the distribution of the signal-to-noise ratio (SNR) at the user terminals. Furthermore, we demonstrate how DMNT can be applied to estimate the long-term throughput for each mobile station, which allows for determining the optimal number of multiplexing order and throughput loss due to the interference nulling.
\end{abstract}

\section{Introduction}

Multiuser MIMO (MU-MIMO) scheme, allowing a base station (BS) to communicate with multiple users simultaneously, provides an opportunity to boost the sum capacity through precoding, even when each user has only one antenna. For example, zero-forcing transmit beamforming (ZFBF) is one of the practical multiuser transmission strategies for MUMIMO systems [1]. By designing one user's beamforming vector to be orthogonal to other selected users' channel vectors, $Z F B F$ can completely eliminate the multiuser interference corresponding to intracell interference in cellular systems. Furthermore, using more transmit antenna can increase the number of users simultaneously served by MUMIMO or enhance the error performance of each link between the BS and user.

Despite the theoretical attractiveness, the capacity gain promised by MIMO techniques has been shown to degrade severely in a multicell environment. To suppress the intercell interference, the authors in [2-5] investigated a coordinated beamforming scheme using multiple antennas at the BS. The achievable rate region of the MISO interference channel, in the case where the full channel information is shared among BSs, was derived in $[2,3]$, with instantaneous and statistical CSI, respectively. Distributed beamforming with a virtual SINR framework was proposed in [4]. The theoretical results in [2-4], however, are limited to only one user in the victim cell. The authors in [5] assumed that the interference experienced by multiple users in the victim cells is suppressed. Some studies on the interference mitigation in the cooperative beamforming for multiuser systems have been studied from the perspective of scheduling issues [6-9]. A low-complexity random beamforming method, which only requires sharing of user indices, has been suggested with analytic throughput expressions [6]. In [7], the authors provided a transmission beamforming scheme for interference nulling with user selection. Also, the reduced complexity algorithms for joint user selection in adaptive coordination scheme were designed in [8]. However, the unrealistic special homogeneous case, in which all users have the same average SNR, is assumed in [8]. 
In [9], we have considered a generalized intercell interference coordination problem and proposed a two-step coordination procedure to choose a cell-edge user and decide the coordination. However, the research in [6-9] was not extended to MUMIMO, that is, only dealing with the multiple users in the serving cell.

In this paper, we analyze the three-dimensional DMNT among the diversity order $D$ (i.e., slope of error performance curve), multiplexing order $L$ (i.e., the number of users simultaneously served by MU-MIMO scheme), and nulling order $N$ (i.e., the number of other cell users subject to zero interference in a victim cell), while providing the victim cell with intercell interference nulling. We consider an interferencefree environment in which the BS in each cell employs a precoding matrix with $N_{T}$ antennas, so as to null the intracell interference while mitigating the intercell interference. It is assumed that all users are equipped with a single antenna. Our contribution is to reveal the fundamental property of performance trade-off, given by $N_{T}=D+L+N-1$, in multicell MU-MIMO subject to intercell and intracell interferencefree conditions. To the best of our knowledge, there has never been any rigorous justification for this particular property in previous works. Note that the current diversity-multiplexingnulling trade-off (DMNT) is quite different from the wellknown diversity-multiplexing trade-off (DMT) that deals with the multiple antenna gain to be achieved simultaneously by any coding scheme (e.g., space-time coding) in the pointto-point MIMO system [10]. Meanwhile, we demonstrate that our DMNT can be applicable to estimating the long-term user throughput, which allows for determining the optimal multiplexing order and throughput loss due to interference nulling.

The rest of this paper is organized as follows. We present some preliminaries for our analysis in Section 2. Section 3 presents a system model and the precoding matrix design under consideration. Our analysis results for the diversitymultiplexing-nulling trade-off are given in Section 4. Section 5 demonstrates how DMNT can be applied to estimate the long-term throughput for each MS. Finally, concluding remarks are given in Section 6.

\section{Preliminaries}

We first introduce some results from previous works, which are useful for our analysis.

Definition 1. Let $\mathbf{x}_{1}, \mathbf{x}_{2}, \ldots, \mathbf{x}_{n}$ be independent complex Gaussian random vectors with zero mean vector (i.e., $E\left\{\mathbf{x}_{i}\right\}=\mathbf{0}_{p}$ ) and identity covariance matrix (i.e., $E\left\{\mathbf{x}_{i} \mathbf{x}_{i}^{H}\right\}=\mathbf{I}_{p}$ ). If $\mathbf{A}=$ $\mathbf{X X}^{H}$, where $\mathbf{X}=\left[\begin{array}{llll}\mathbf{x}_{1} & \mathbf{x}_{2} & \cdots & \mathbf{x}_{n}\end{array}\right]$ is the $p \times n$ matrix, then $\mathbf{A}$ is said to have a complex Wishart distribution with $n$ degrees of freedom [11], that is, $\mathbf{A} \sim W_{p}(n)$.

By using $n$ vectors, we may define the matrix $\mathbf{A}$ as

$$
\mathbf{A}=\mathbf{X} \mathbf{X}^{H}=\sum_{i=1}^{n} \mathbf{x}_{i} \mathbf{x}_{i}^{H} .
$$

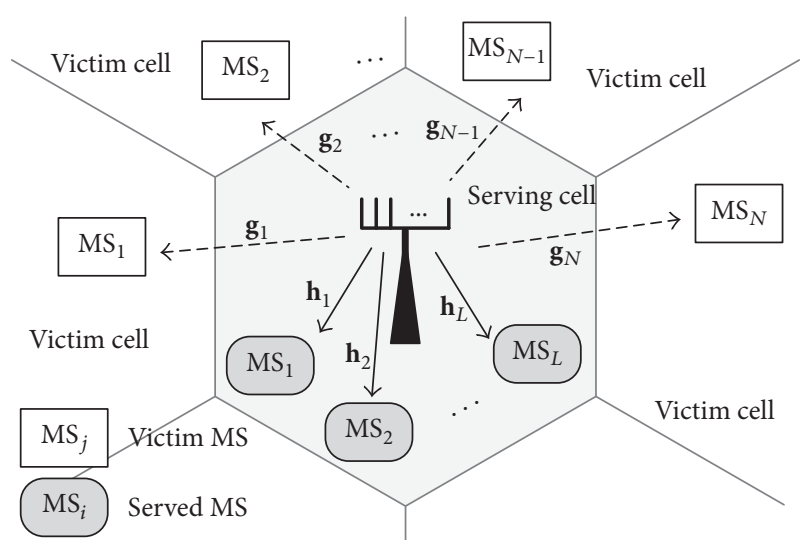

FIGURE 1: System model: serving cell and victim cells.

The probability density function of $\mathbf{A}$ for $n \geq p$ is given as

$$
p_{W}(\mathbf{A})=\frac{\exp (-\operatorname{tr}(\mathbf{A}))(\operatorname{det} \mathbf{A})^{n-p}}{\pi^{p(p-1) / 2} \prod_{j=1}^{p} \Gamma(n-j+1)},
$$

where $\Gamma(z)=\int_{0}^{\infty} e^{-t} t^{z-1} d t$. If $p=1$, that is, $\mathbf{A}$ is defined as a random variable, then it has a Chi-squared distribution with $2 n$ degrees of freedom, that is, $\mathbf{A} \sim \chi^{2}(2 n)$. This result immediately follows by substituting $p=1$ into the probability density function (pdf) of the Wishart distribution.

Lemma 2. If $\mathbf{A} \sim W_{p}(n)$ and $\mathbf{A}$ is partitioned as

$$
\mathbf{A}=\left[\begin{array}{ll}
\mathbf{A}_{11} & \mathbf{A}_{12} \\
\mathbf{A}_{21} & \mathbf{A}_{22}
\end{array}\right],
$$

where $\mathbf{A}_{11}$ is $k \times k$ and the Schur complement of block $\mathbf{A}_{22}$ is also a Wishart matrix with a distribution of $W_{k}(n-p+k)$. In [12], the Schur complement of block $\mathbf{A}_{22}$ is given as

$$
\widetilde{\mathbf{A}}_{11}=\mathbf{A}_{11}-\mathbf{A}_{12} \mathbf{A}_{22}^{-1} \mathbf{A}_{21} \text {. }
$$

Proof. See proof of Theorem 3.2.10 in [13].

\section{Signal Model and Precoding Matrix Design}

We consider MU-MIMO downlink systems in which the BS serves a set of selected mobile stations (MSs) simultaneously in a serving cell, while imparting interference to the MSs in victim cells, as illustrated in Figure 1. We assume that there are $K$ and $N$ MSs in the serving cell and victim cells, respectively. Let $\delta$ be a subset of indices for users that are intended for transmission by the BS $(\mathcal{S} \subset\{1,2, \ldots, K\},|\mathcal{S}|=L \leq K)$. The user set $\mathcal{S}$ is dynamically selected by a scheduler in the BS. At the serving cell, we design a wireless link equipped with $N_{T}$ transmit antennas at the BS and a single receive antenna at each MS. The MSs in the victim cells also employ a single receive antenna and do not perform any type of interference mitigation. Let us denote $\mathbf{h}_{k}$ and $\mathbf{w}_{k}$ as $N_{T} \times 1$ complex Gaussian channel vector and beamforming vector for the $k$ th MS, respectively. For a subset $\mathcal{S}$, we define $\mathbf{H}(\mathcal{S})=$ 
$\left[\begin{array}{lll}\mathbf{h}_{1} & \cdots & \mathbf{h}_{L}\end{array}\right]$ and $\mathbf{W}(\mathcal{S})=\left[\begin{array}{lll}\mathbf{w}_{1} & \cdots & \mathbf{w}_{L}\end{array}\right]$. The received signal $y_{l}$ at the $l$ th MS in the subset $\mathcal{S}$ is represented by

$$
y_{l}=\mathbf{h}_{l}^{H} \mathbf{w}_{l} s_{l}+\sum_{j=1, j \neq l}^{L} \mathbf{h}_{l}^{H} \mathbf{w}_{j} s_{j}+n_{l}, \quad l=1,2, \ldots, L \in \mathcal{S}
$$

where $s_{l}$ and $n_{l}$ are the data symbol and the Additive White Gaussian Noise (AWGN) with variance of $N_{0}$, respectively. We impose average power constraints, $\left\|\mathbf{w}_{l}\right\|^{2}=1$ and $E\left\{\left|s_{l}\right|^{2}\right\}=E_{s}$. The received signals in (5) are rewritten by the aggregated received signal vector $\mathbf{y}$ as

$$
\mathbf{y}=\mathbf{H}^{H} \mathbf{W} \mathbf{s}+\mathbf{n}
$$

where $\mathbf{s}=\left[\begin{array}{lll}s_{1} & \cdots & s_{L}\end{array}\right]^{T}$ and $\mathbf{n}=\left[\begin{array}{lll}n_{1} & \cdots & n_{L}\end{array}\right]^{T}$. Let $\mathbf{g}_{j}$ denote an $N_{T} \times 1$ channel vector from the serving BS to the $j$ th MS in the victim cell. Note that $N$ MSs in the victim cells are those subject to intercell interference. In our current system model in Figure 1, N MSs in the virtual victim cell can be considered as those multiplexed with $L$ MSs in the serving cell while satisfying the intercell interference-free condition. For the MSs in the victim cell, the aggregated received signal vector $\tilde{\mathbf{y}}$ is given by

$$
\widetilde{\mathbf{y}}=\mathbf{G}^{H} \mathbf{W} \mathbf{s},
$$

where $\mathbf{G}=\left[\begin{array}{lll}\mathbf{g}_{1} & \cdots & \mathbf{g}_{N}\end{array}\right]$, which can be known to the serving BS by a sounding signal [14]. Note that a desired signal of the victim MS is not represented in (7); that is, $\widetilde{\mathbf{y}}$ is just an intercell interference vector for the victim MSs, which would be controlled by the BS in the serving cell.

Our proposed precoding matrix design focuses on achieving the interference-free communication for both interferences from the other cell and other user signal from the serving BS. First, the intercell interference-free condition is given as

$$
\widetilde{\mathbf{y}}=\mathbf{G}^{H} \mathbf{W} \mathbf{s}=\mathbf{0}_{N},
$$

where $\mathbf{0}_{N}$ denotes an $N \times 1$ column vector with all-zero elements. Our objective is to rigorously show how much the spatial degrees of freedom are lost in this situation. Note that the intercell interference-free condition (8) leads to the following proposition:

Proposition 3. To satisfy the intercell interference-free condition, Ws should lie in the null space of $\mathbf{G}$, that is, the orthogonal complement of the subspace $\mathscr{G}$ spanned by column vectors $\mathbf{g}_{1}, \mathbf{g}_{2}, \ldots, \mathbf{g}_{N}$ of $\mathbf{G}$.

From Proposition 3, the precoding matrix $\mathbf{W}$ can be a cascade of matrices, $\mathbf{W}^{\text {inter }}$ and $\mathbf{W}^{\text {intra }}$, that is, $\mathbf{W}=$ $\mathbf{W}^{\text {inter }} \mathbf{W}^{\text {intra }}$, where $\mathbf{W}^{\text {inter }}$ eliminates the intercell interference by adopting a projection matrix onto the orthogonal complement (PMOC) of $\mathscr{G}$. If $\mathbf{W}^{\text {inter }}$ is a PMOC of $\mathscr{G}, \mathbf{W s}$ lies in the null space of $\mathbf{G}$, regardless of $\mathbf{W}^{\text {intra }}$. Therefore, we may design two precoding matrices, $\mathbf{W}^{\text {inter }}$ and $\mathbf{W}^{\text {intra }}$, independently, so as to meet each design constraint.
To produce a PMOC of $\mathscr{G}$, consider the following $\mathrm{QR}$ decomposition of $\mathbf{G}$ :

$$
\mathbf{G}=\mathbf{Q R}
$$

where $\mathbf{Q}$ is an $N_{T} \times N_{T}$ unitary matrix and $\mathbf{R}$ is an $N_{T} \times N$ upper triangular matrix. As the bottom $\left(N_{T}-N\right)$ rows of $\mathbf{R}$ consist of entire zeroes, it is often useful to partition $\mathbf{Q}$ and $\mathbf{R}$ as follows:

$$
\mathbf{G}=\mathbf{Q R}=\mathbf{Q}\left[\begin{array}{c}
\mathbf{R}_{1} \\
\mathbf{0}
\end{array}\right]=\left[\begin{array}{ll}
\mathbf{Q}_{1} & \mathbf{Q}_{2}
\end{array}\right]\left[\begin{array}{c}
\mathbf{R}_{1} \\
\mathbf{0}
\end{array}\right]=\mathbf{Q}_{1} \mathbf{R}_{1},
$$

where $\mathbf{R}_{1}$ is an $N \times N$ upper triangular matrix, $\mathbf{Q}_{1}$ is $N_{T} \times N$ orthogonal matrix, $\mathbf{Q}_{2}$ is $N_{T} \times\left(N_{T}-N\right)$, and both $\mathbf{Q}_{1}$ and $\mathbf{Q}_{2}$ have orthogonal columns. Let $\mathbf{P}_{\mathbf{G}}^{\perp}$ denote a PMOC of $\mathscr{G}$. Then, we can obtain $\mathbf{P}_{\mathbf{G}}^{\perp}$ by using $\mathbf{Q}_{1}$ or $\mathbf{Q}_{2}$ as follows:

$$
\mathbf{P}_{\mathbf{G}}^{\perp}=\mathbf{I}_{N_{T}}-\mathbf{Q}_{1} \mathbf{Q}_{1}^{H}=\mathbf{Q}_{2} \mathbf{Q}_{2}^{H} .
$$

Using $\mathbf{Q}_{1}=\mathbf{G R}_{1}^{-1}$ and $\mathbf{R}_{1}=\mathbf{Q}_{1}^{H} \mathbf{G}$, alternative form can be represented as

$$
\mathbf{P}_{\mathbf{G}}^{\perp}=\mathbf{I}_{N_{T}}-\mathbf{Q}_{1} \mathbf{Q}_{1}^{H}=\mathbf{I}_{N_{T}}-\mathbf{G}\left(\mathbf{G}^{H} \mathbf{G}\right)^{-1} \mathbf{G}^{H}
$$

Let us now design $\mathbf{W}^{\text {intra }}=\left[\begin{array}{llll}\mathbf{w}_{1}^{\text {intra }} & \cdots & \mathbf{w}_{L}^{\text {intra }}\end{array}\right]$, whose purpose is to avoid interuser interference. Denoting $\widetilde{\mathbf{H}}^{H}=$ $\mathbf{H}^{H} \mathbf{P}_{\mathbf{G}}^{\perp}=\left[\begin{array}{llll}\widetilde{\mathbf{h}}_{1} & \widetilde{\mathbf{h}}_{2} & \cdots & \widetilde{\mathbf{h}}_{L}\end{array}\right]^{H}$ for ZFBF, beamforming vectors are selected such that they satisfy the zero-interference condition $\widetilde{\mathbf{h}}_{k}^{H} \mathbf{w}_{l}^{\text {intra }}=0$ for $k \neq l$; that is, the beamforming vector for user $l$ lies in the null space spanned by $\left\{\widetilde{\mathbf{h}}_{k}, \forall k \neq l\right\}$. One easy choice of the precoding matrix that gives zero interuser interference is the pseudoinverse; that is,

$$
\widetilde{\mathbf{W}}=\widetilde{\mathbf{H}}\left(\widetilde{\mathbf{H}}^{H} \widetilde{\mathbf{H}}\right)^{-1}=\left[\begin{array}{llll}
\widetilde{\mathbf{w}}_{1} & \widetilde{\mathbf{w}}_{2} & \cdots & \widetilde{\mathbf{w}}_{L}
\end{array}\right] .
$$

The precoding matrix $\mathbf{W}^{\text {intra }}$ is formed by the unitnormalized columns of $\widetilde{\mathbf{W}}$; that is, $\mathbf{w}_{l}^{\text {intra }}=\widetilde{\mathbf{w}}_{l} /\left\|\mathbf{P}_{\mathbf{G}}^{\perp} \widetilde{\mathbf{w}}_{l}\right\|$. In matrix form, $\mathbf{W}^{\text {intra }}$ is given by

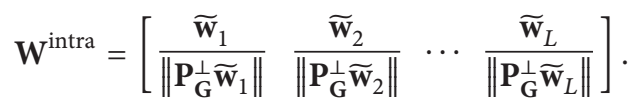

Finally, the aggregated received signal vector $\mathbf{y}$ is given by

$$
\begin{aligned}
\mathbf{y} & =\widetilde{\mathbf{H}}^{H} \mathbf{W}^{\text {intra }} \mathbf{s}+\mathbf{n} \\
& =\widetilde{\mathbf{H}}^{H}\left[\frac{\widetilde{\mathbf{w}}_{1}}{\left\|\mathbf{P}_{\mathbf{G}}^{\perp} \widetilde{\mathbf{w}}_{1}\right\|} \cdots \frac{\widetilde{\mathbf{w}}_{L}}{\left\|\mathbf{P}_{\mathbf{G}}^{\perp} \widetilde{\mathbf{w}}_{L}\right\|}\right] \mathbf{s}+\mathbf{n} \\
& =\widetilde{\mathbf{H}}^{H} \widetilde{\mathbf{W}} \cdot \operatorname{diag}\left(\frac{1}{\left\|\mathbf{P}_{\mathbf{G}}^{\perp} \widetilde{\mathbf{w}}_{1}\right\|}, \ldots, \frac{1}{\left\|\mathbf{P}_{\mathbf{G}}^{\perp} \widetilde{\mathbf{w}}_{L}\right\|}\right) \mathbf{s}+\mathbf{n} .
\end{aligned}
$$

Since $\widetilde{\mathbf{W}}=\widetilde{\mathbf{H}}\left(\widetilde{\mathbf{H}}^{H} \widetilde{\mathbf{H}}\right)^{-1}$, the received signal $y_{l}$ is given as

$$
y_{l}=\frac{s_{l}}{\left\|\mathbf{P}_{\mathbf{G}}^{\perp} \widetilde{\mathbf{w}}_{l}\right\|}+n_{l}
$$




\section{Analysis of Diversity-Multiplexing-Nulling Trade-Off (DMNT)}

The effective SNR at the $l$ th MS is given by $\gamma_{l}=\bar{\gamma}_{l} /\left\|\mathbf{P}_{\mathbf{G}}^{\perp} \widetilde{\mathbf{w}}_{l}\right\|^{2}$, where $\bar{\gamma}_{l}=E_{s} / N_{0}$ denotes the average SNR. As $\mathbf{P}_{\mathbf{G}}^{\perp} \widetilde{\mathbf{W}}=$ $\left[\begin{array}{llll}\mathbf{P}_{\mathbf{G}}^{\perp} \widetilde{\mathbf{w}}_{1} & \mathbf{P}_{\mathrm{G}}^{\perp} \widetilde{\mathbf{w}}_{2} & \cdots & \mathbf{P}_{\mathbf{G}}^{\perp} \widetilde{\mathbf{w}}_{L}\end{array}\right]$, we have

$$
\left\|\mathbf{P}_{\mathbf{G}}^{\perp} \widetilde{\mathbf{w}}_{l}\right\|^{2}=\left[\left(\mathbf{P}_{\mathbf{G}}^{\perp} \widetilde{\mathbf{W}}\right)^{H}\left(\mathbf{P}_{\mathbf{G}}^{\perp} \widetilde{\mathbf{W}}\right)\right]_{l l},
$$

where $[\mathbf{X}]_{l l}$ denotes an $(l, l)$-diagonal element of $\mathbf{X}$. Using (17) and $\widetilde{\mathbf{W}}=\widetilde{\mathbf{H}}\left(\widetilde{\mathbf{H}}^{H} \widetilde{\mathbf{H}}\right)^{-1}$, we have

$$
\gamma_{l}=\frac{\bar{\gamma}_{l}}{\left[\left(\widetilde{\mathbf{H}}^{H} \widetilde{\mathbf{H}}\right)^{-1} \widetilde{\mathbf{H}}^{H} \mathbf{P}_{\mathbf{G}}^{\perp H} \mathbf{P}_{\mathbf{G}}^{\perp} \widetilde{\mathbf{H}}\left(\widetilde{\mathbf{H}}^{H} \widetilde{\mathbf{H}}\right)^{-1}\right]_{l l}} .
$$

Now, defining $\overline{\mathbf{H}}:=\widetilde{\mathbf{H}}^{H}$, we have

$$
\gamma_{l}=\frac{\bar{\gamma}_{l}}{\left[\left(\overline{\mathbf{H}} \overline{\mathbf{H}}^{H}\right)^{-1} \overline{\mathbf{H}} \mathbf{P}_{\mathbf{G}}^{\perp H} \mathbf{P}_{\mathbf{G}}^{\perp} \overline{\mathbf{H}}^{H}\left(\overline{\mathbf{H}} \overline{\mathbf{H}}^{H}\right)^{-1}\right]_{l l}} .
$$

Meanwhile, $\overline{\mathbf{H}}=\mathbf{H}^{H} \mathbf{P}_{\mathbf{G}}^{\perp}$ from $\widetilde{\mathbf{H}}^{H}=\mathbf{H}^{H} \mathbf{P}_{\mathbf{G}}^{\perp}$ and $\overline{\mathbf{H}}=\widetilde{\mathbf{H}}^{H}$, which gives

$$
\begin{aligned}
\overline{\mathbf{H}} \mathbf{P}_{\mathbf{G}}^{\perp H} \mathbf{P}_{\mathbf{G}}^{\perp} \overline{\mathbf{H}}^{H} & =\mathbf{H}^{H} \mathbf{P}_{\mathbf{G}}^{\perp} \mathbf{P}_{\mathbf{G}}^{\perp H} \mathbf{P}_{\mathbf{G}}^{\perp} \mathbf{P}_{\mathbf{G}}^{\perp H} \mathbf{H}=\mathbf{H}^{H} \mathbf{P}_{\mathbf{G}}^{\perp} \mathbf{P}_{\mathbf{G}}^{\perp H} \mathbf{H} \\
& =\overline{\mathbf{H}} \overline{\mathbf{H}}^{H} .
\end{aligned}
$$

The last step follows from the properties of the projection matrix; that is, $\mathbf{P}_{\mathbf{G}}^{\perp H}=\mathbf{P}_{\mathbf{G}}^{\perp}$ and $\mathbf{P}_{\mathbf{G}}^{\perp 2}=\mathbf{P}_{\mathbf{G}}^{\perp}$. Finally, we have

$$
\gamma_{l}=\frac{\bar{\gamma}_{l}}{\left[\left(\overline{\mathbf{H}} \overline{\mathbf{H}}^{H}\right)^{-1}\right]_{l l}}=\frac{\bar{\gamma}_{l}}{\left[\left(\widetilde{\mathbf{H}}^{H} \widetilde{\mathbf{H}}\right)^{-1}\right]_{l l}} \quad\left(\because \widetilde{\mathbf{H}}=\overline{\mathbf{H}}^{H}\right) \text {. }
$$

Let $\mathbf{Z}=\widetilde{\mathbf{H}}^{H} \widetilde{\mathbf{H}}$. Without loss of generality, we obtain the SNR for $l=1$ as follows:

$$
\gamma_{1}=\frac{\bar{\gamma}_{1}}{\left[\mathbf{Z}^{-1}\right]_{11}}=\frac{\bar{\gamma}_{1} \operatorname{det}(\mathbf{Z})}{\operatorname{adj}(\mathbf{Z})_{11}} .
$$

The last equality in (22) follows from Cramer's rule [14] and the $(i, j)$-element of $\operatorname{adj}(\mathbf{Z})$ is given by

$$
\operatorname{adj}(\mathbf{Z})_{i j}=(-1)^{i+j} \operatorname{det}\left(\mathbf{Z}_{\overline{j i}}\right)
$$

where $\mathbf{Z}_{\overline{j i}}$ is the $(L-1) \times(L-1)$ matrix formed by deleting the $j$ th row and $i$ th column of $\mathbf{Z}$. When $\mathbf{Z}$ is partitioned as

$$
\mathbf{Z}=\left[\begin{array}{ll}
z_{11} & \mathbf{z}_{12} \\
\mathbf{z}_{21} & \mathbf{Z}_{22}
\end{array}\right]
$$

$\operatorname{adj}(\mathbf{Z})_{11}=\operatorname{det}\left(\mathbf{Z}_{22}\right)$ from (23). Thus, (22) can be expressed as

$$
\gamma_{1}=\frac{\bar{\gamma}_{1} \operatorname{det}(\mathbf{Z})}{\operatorname{det}\left(\mathbf{Z}_{22}\right)}
$$

To proceed, we use the following property:

$$
\operatorname{det}\left(\left[\begin{array}{ll}
\mathbf{A} & \mathbf{B} \\
\mathbf{C} & \mathbf{D}
\end{array}\right]\right)=\operatorname{det}(\mathbf{D}) \operatorname{det}\left(\mathbf{A}-\mathbf{B D}^{-1} \mathbf{C}\right) \text {. }
$$

This in turn yields

$$
\gamma_{1}=\bar{\gamma}_{1} \operatorname{det}\left(z_{11}-\mathbf{z}_{12} \mathbf{Z}_{22}^{-1} \mathbf{z}_{21}\right)=\bar{\gamma}_{1} \tilde{z}_{11}
$$

where $\widetilde{z}_{11}=z_{11}-\mathbf{z}_{12} \mathbf{Z}_{22}^{-1} \mathbf{z}_{21}$; that is, $\widetilde{z}_{11}$ is the Schur complement of block $\mathbf{Z}_{22}$. We can show that the distribution of $\gamma_{1}$ is given by the following lemma, which will be useful for analyzing the DMNT.

Lemma 4. $\gamma_{1}$ has a chi-squared distribution with $2\left(N_{T}-N-\right.$ $L+1$ ) degrees of freedom.

Proof. See Appendix.

It is well-known that the diversity order (corresponding to a slope of an error performance curve) is $n$ when the SNR is distributed as a chi-squared distribution with $2 n$ degrees of freedom [15]. By Lemma 4, therefore, the diversity order of the interference-free MU-MIMO system under consideration is given by $N_{T}-N-L+1$, which leads to the following result for the DMNT.

Theorem 5. Let $N, L$, and D denote the nulling order, multiplexing order, and diversity order for the downlink BS in the multicell MU-MIMO system. Assuming a BS equipped with $N_{T}$ antennas and MSs equipped with a single antenna, the trade-off among the nulling order, multiplexing order, and diversity order for the interference-free condition is given by

$$
N_{T}=D+L+N-1 \text {. }
$$

\section{Application of DMNT: Asymptotic Throughput Analysis}

In this section, DMNT in Theorem 5 can be applied to estimate the long-term throughput for each MS when an opportunistic ZFBF scheduling is applied. It deals with an asymptotic throughput analysis, from which the optimal number of multiplexing order and the throughput loss due to the interference nulling can be achieved. In fact, it will be a useful analytical framework that can predict how DMN must be traded off so as to maximize the cell throughput or to maintain a required level of cell throughput.

Let $\gamma_{l}(\mathcal{S})$ represent the effective SNR for the $l$ th user when $\mathcal{S}$ is a set of the selected users that are transmitted at the same time, as defined in Section 3. The objective of the opportunistic scheduling is to determine a subset of users, $\mathcal{S}$, such that the sum rate is maximized; that is,

$$
\mathcal{S}^{*}=\underset{\mathcal{S}}{\arg \max } \sum_{l=1}^{L} R_{l}(\mathcal{S}),
$$

where $R_{l}(\mathcal{S})=\log _{2}\left(1+\gamma_{l}(\mathcal{S})\right.$ ). Assuming $L=2$ (scalable to $L>2$ with the same principle), the long-term throughput $\bar{R}_{k}$ is given by

$$
\bar{R}_{k}=\operatorname{Pr}\left(k \in \mathcal{S}^{*}\right) E\left\{R_{k}\left(\mathcal{S}^{*}\right) \mid k \in \mathcal{S}^{*}\right\} .
$$


Since the channel vectors have independent and identical distribution, $\operatorname{Pr}\left(\mathcal{S}^{*}=\{k, 1\}\right)=(1 /(K-1)) \cdot \operatorname{Pr}\left(k \in \mathcal{S}^{*}\right)$ and thus (30) can be expressed as

$$
\begin{aligned}
& \bar{R}_{k} \\
& =(K-1) \operatorname{Pr}\left(\mathcal{S}^{*}=\{k, 1\}\right) E\left\{R_{k}\left(\mathcal{S}^{*}\right) \mid \mathcal{S}^{*}=\{k, 1\}\right\} .
\end{aligned}
$$

Furthermore, since $\operatorname{Pr}\left(\mathcal{S}^{*}=\{k, 1\}\right)=1 / K(K-1)$, we have

$$
\begin{aligned}
\bar{R}_{k} & =\frac{1}{K} E\left\{R_{k}\left(\mathcal{S}^{*}\right) \mid \mathcal{S}^{*}=\{k, 1\}\right\}=\frac{1}{K} \\
& \cdot E\left\{R_{k}\left(\mathcal{S}^{*}\right) \mid R_{k}(\{k, 1\})+R_{1}(\{k, 1\}) \geq R_{i}(\{i, 1\})\right. \\
& \left.+R_{1}(\{i, 1\}), \forall i \neq k\right\} .
\end{aligned}
$$

As $K \rightarrow \infty$, there will be multiple MSs (i.e., $k$ th MS and others) who have near orthogonal channel vector with respect to MS 1 . Thus, we can assume that $R_{1}(\{k, 1\}) \approx R_{1}(\{i, 1\})$ for sufficiently large $K$, reducing (32) into

$$
\begin{aligned}
\bar{R}_{k} & \approx \frac{1}{K} E\left\{R_{k}\left(S^{*}\right) \mid R_{k}(\{k, 1\}) \geq R_{i}(\{i, 1\}), \forall i \neq k\right\} \\
& =\frac{1}{K} E\left\{R_{k}\left(S^{*}\right) \mid \gamma_{k} \geq \gamma_{i}, \forall i \neq k\right\} .
\end{aligned}
$$

Equation (33) implies that, for large $K$, the long-term throughput can be obtained by a single user scheduling problem in which each MS has a diversity order of $\left(N_{T}-\right.$ $N-L+1)$, as dictated by Theorem 5 and therefore, it follows the chi-squared distribution with $2\left(N_{T}-N-L+1\right)$ degrees of freedom. We note that the long-term throughput for the single user scheduling can be obtained by using the extreme value theory [16]. Based on the results in [16], the analytic throughput result is obtained as

$$
\begin{aligned}
\bar{R}_{k} & =\frac{1}{K} \log _{2}\left(1+F_{\gamma_{k}}^{-1}\left(\frac{K-1}{K}\right)\right)+\frac{1}{K} \\
& \times E_{0}\left(\log _{2}\left(1+F_{\gamma_{k}}^{-1}\left(\frac{K \cdot e-1}{K \cdot e}\right)\right)\right. \\
& \left.-\log _{2}\left(1+F_{\gamma_{k}}^{-1}\left(\frac{K-1}{K}\right)\right)\right),
\end{aligned}
$$

where $F_{\gamma_{k}}(\gamma)$ is the CDF of $\gamma_{k}$ in Lemma 4 and $E_{0}=0.5772$, which is the Euler constant [17].

In order to validate the accuracy of our asymptotic throughput analysis, we simulate the case with $N_{T}=8, N=$ 2 , and $L=2$, and uniformly distributed $30 \mathrm{MSs}$; that is, $K=30$. Since all users have the different average SNR in the simulation, the different average throughput is observed for individual user as shown in Figure 2. We find that throughput measurement for each MS is acceptably close to the analytical result. Furthermore, the average cell throughput $\sum_{k} \bar{R}_{k}$ can be determined by the asymptotic throughput analysis for $N_{T}=$ 16 and $K=100$, as shown in Figures 3 and 4. Figure 3 shows that there exists a multiplexing order to maximize the cell throughput for the given nulling order, demonstrating the optimal number of multiplexing order and the throughput loss due to the interference nulling. For example,

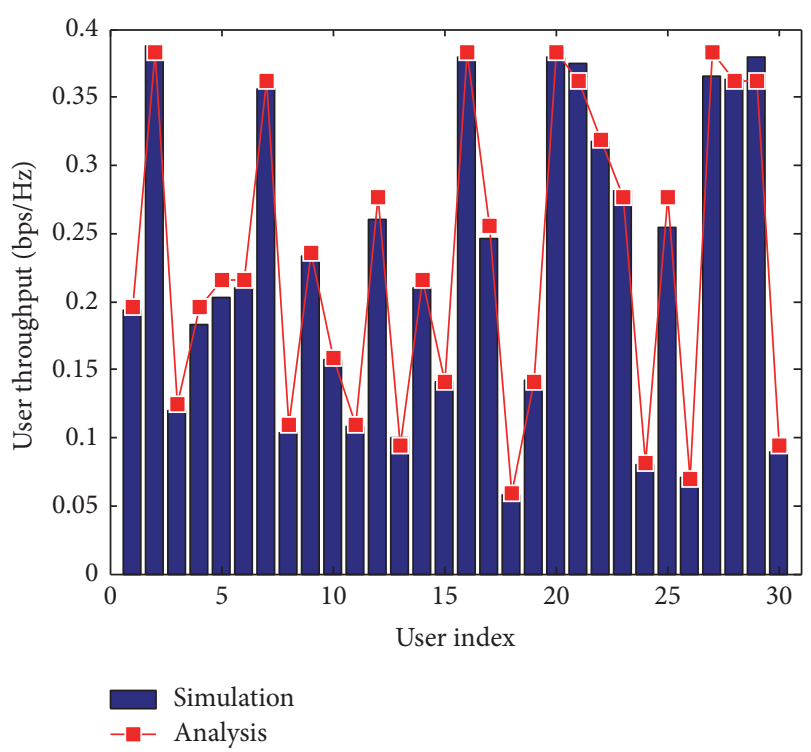

FIGURE 2: Throughput estimation for each MS: analysis versus simulation.

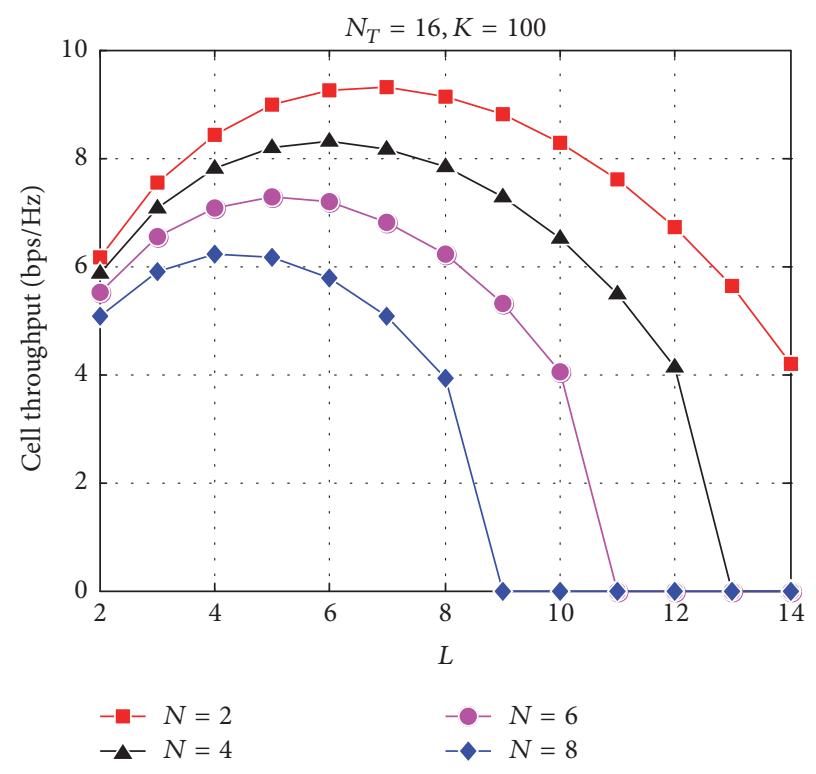

FIGURE 3: Cell throughput: diversity order $(D)$ versus multiplexing order $(L)$.

when two other cell users are nulled (i.e., $N=2$ ), the optimal number of multiplexing gains is 7; that is, 7 active users must be served in the reference cell $(L=7)$. Furthermore, it is obvious from Figure 3 that the average cell throughput is reduced as increasing the nulling order. The current analysis implies that the multiplexing order should be adjusted adaptively for the different nulling order. Furthermore, it is observed that the cell throughput gained by reducing the interference-nulling effect subject to the same diversity gain becomes more conspicuous as the diversity order increases; that is, the multiplexing order decreases. Figure 4 shows the same observation for varying the nulling order for 


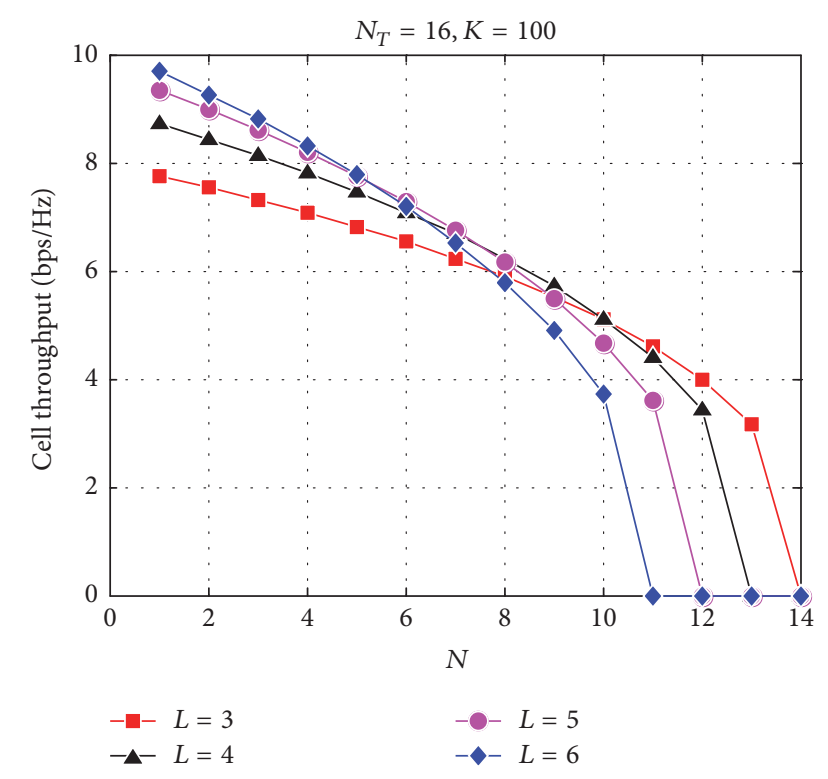

FIGURE 4: Cell throughput: diversity order $(D)$ versus nulling order $(N)$.

the given multiplexing order. Unlike Figure 3, there is no optimal operating point between $N$ and $D$. Furthermore, it is observed that the multiplexing gain contributes to the cell throughput differently, depending on the nulling order. For example, spatial multiplexing is more important than diversity in improving the cell throughput if the nulling order is small. Otherwise, diversity gain is more contributing to the cell throughput than multiplexing gain. Meanwhile, the maximum nulling order $N$ that is required to maintain the minimum target cell throughput can be determined by the curves in Figure 4.

\section{Conclusion}

In this paper, we have investigated a fundamental property of performance trade-off in the multicell multiuser multiple-input multiple-output (MU-MIMO) system when the intercell and intracell interference-free conditions must be satisfied. Assuming a BS equipped with $N_{T}$ antennas and MSs equipped with a single antenna, the trade-off among the nulling order $(N)$, multiplexing order $(L)$, and diversity order $(D)$ for the interfere-free condition is given by $N_{T}=D+$ $L+N-1$. By characterizing the three-dimensional diversitymultiplexing-nulling trade-off (DMNT), our analysis provides a quantitative framework for dealing with the intercell interference coordination in a multicell MU-MIMO system. Finally, we have demonstrated that our DMNT result can be used to perform an asymptotic throughput analysis, which predicts how DMN must be traded off so as to maximize the cell throughput or to maintain a required level of cell throughput in the system. The overall system performance can be optimized by selecting a set of users and the precoders at the same time. As the joint optimization involves enormous complexity, joint optimization seems to be unrealistic in practice. It will be worth investigating the suboptimal approach that can be implemented in practice. However, user selection and codebook design subject to the limited feedback is beyond our scope in this paper. In other words, even if the overall system performance is governed by user selection and codebook design, along with channel estimation error, we aimed at demonstrating Diversity-Multiplexing-Nulling Trade-off (DMN) as an ideal performance characteristic, which would play a fundamental design guideline.

The interference-free environment (realized through the interference-nulling effect) may not be straightforward to achieve in practice. Since we assume that a full CSI is available at BS, an interesting venue for future work is to design a limited feedback system and, furthermore, to analyze the performance subject to channel estimation error. Whereas the current asymptotic analysis illustrates throughput of the reference cell only, we may need a multicell coordination framework in which all neighboring cells are coordinated to determine the optimal DMNT for the overall throughput maximization in practice.

\section{Appendix}

\section{Proof of Lemma 4}

Since $\widetilde{\mathbf{H}}^{H}=\mathbf{H}^{H}\left(\mathbf{I}_{N_{T}}-\mathbf{Q Q} \mathbf{Q}^{H}\right), \mathbf{Z}$ can be expressed as

$$
\mathbf{Z}=\mathbf{H}^{H}\left(\mathbf{I}_{N_{T}}-\mathbf{Q}_{1} \mathbf{Q}_{1}^{H}\right) \mathbf{H} .
$$

In (A.1), $\mathbf{Q}_{1} \mathbf{Q}_{1}^{H}$ can be eigen-decomposed as $\mathbf{Q}_{1} \mathbf{Q}_{1}^{H}=$ $\mathbf{X} \boldsymbol{\Sigma} \mathbf{X}^{H}$ where $\mathbf{X}$ is a unitary matrix and $\boldsymbol{\Sigma}$ is the diagonal matrix whose diagonal elements are the corresponding eigenvalues. Using $\mathbf{Q} \mathbf{Q}^{H}=\mathbf{X} \boldsymbol{\Sigma} \mathbf{X}^{H}$, (A.1) can be expressed as

$$
\begin{aligned}
\mathbf{Z} & =\widehat{\mathbf{H}}^{H}\left(\mathbf{I}_{N_{T}}-\mathbf{\Sigma}\right) \widehat{\mathbf{H}} \\
& =\left[\begin{array}{lll}
\widehat{\mathbf{h}}_{1} & \cdots & \widehat{\mathbf{h}}_{N_{T}}
\end{array}\right]\left(\mathbf{I}_{N_{T}}-\boldsymbol{\Sigma}\right)\left[\begin{array}{lll}
\widehat{\mathbf{h}}_{1} & \cdots & \widehat{\mathbf{h}}_{N_{T}}
\end{array}\right]^{H} .
\end{aligned}
$$

Given the orthogonality of $\mathbf{X}, \mathbf{X}^{H} \mathbf{H}$ is also an i.i.d. complex Gaussian channel matrix. Defining $\widehat{\mathbf{H}}=\mathbf{X}^{H} \mathbf{H}=$ $\left[\begin{array}{llll}\widehat{\mathbf{h}}_{1} & \widehat{\mathbf{h}}_{2} & \cdots & \widehat{\mathbf{h}}_{N_{T}}\end{array}\right]^{H}$, we have

$$
\mathbf{Z}=\widehat{\mathbf{H}}^{H}\left(\mathbf{I}_{N_{T}}-\Sigma\right) \widehat{\mathbf{H}}
$$

Meanwhile, $\mathbf{Q}_{1}$ consists of $N$ orthonormal vectors. In other words, $N$ eigenvalues of $\mathbf{Q}_{1} \mathbf{Q}_{1}^{H}$ have a value of 1, while the others are zero. Without loss of generality, we assume that the diagonal entries are sorted in increasing order. In matrix form, $\boldsymbol{\Sigma}$ is given by

$$
\boldsymbol{\Sigma}=\operatorname{diag}(0, \ldots, 0, \underbrace{1, \ldots, 1}_{\text {length } N}) .
$$

Applying this to (A.3), we have

$$
\begin{aligned}
\mathbf{Z} & =\left[\begin{array}{llllll}
\widehat{\mathbf{h}}_{1} & \cdots & \widehat{\mathbf{h}}_{N_{T}-N} & \mathbf{0} & \cdots & \mathbf{0}
\end{array}\right]\left[\begin{array}{lll}
\widehat{\mathbf{h}}_{1} & \cdots & \widehat{\mathbf{h}}_{N_{T}}
\end{array}\right]^{H} \\
& =\sum_{i=1}^{N_{T}-N} \widehat{\mathbf{h}}_{i} \widehat{\mathbf{h}}_{i}^{H} .
\end{aligned}
$$


From the definition of the Wishart distribution in (1), we conclude that $\mathbf{Z}$ has a Wishart distribution with $\left(N_{T}-N\right)$ degrees of freedom. Furthermore, we also find by Lemma 2 that $\widetilde{z}_{11}$ in (27) has a Wishart distribution. Therefore, it is clear that $\widetilde{z}_{11} \sim W_{k}\left(N_{T}-N-L+1\right)$. By Definition 1 , therefore, $\widetilde{z}_{11}$ is a chi-squared random variable with $2\left(N_{T}-N-L+1\right)$ degrees of freedom.

\section{Conflicts of Interest}

The authors declare that there are no conflicts of interest regarding the publication of this article.

\section{Acknowledgments}

This work was supported in part by Institute for Information \& Communications Technology Promotion (IITP) grant funded by the Korea government (MSIT) (no. 2014-0-00282, Development of $5 \mathrm{G}$ Mobile Communication Technologies for Hyperconnected smart services) and in part by the Brain Korea 21 Plus Project in 2017.

\section{References}

[1] T. Yoo and A. Goldsmith, "On the optimality of multiantenna broadcast scheduling using zero-forcing beamforming," IEEE Journal on Selected Areas in Communications, vol. 24, no. 3, pp. 528-541, 2006.

[2] E. A. Jorswieck, E. G. Larsson, and D. Danev, "Complete characterization of the Pareto boundary for the MISO interference channel," IEEE Transactions on Signal Processing, vol. 56, no. 10, part 2, pp. 5292-5296, 2008.

[3] J. Lindblom, E. Karipidis, and E. G. Larsson, "Selfishness and altruism on the MISO interference channel: the case of partial transmitter CSI," IEEE Communications Letters, vol. 13, no. 9, pp. 667-669, 2009.

[4] R. Zakhour and D. Gesbert, "Coordination on the MISO interference channel using the virtual SINR framework," in Proceedings of the International ITG Workshop on Smart Antennas, Berlin, Germany, February 2009.

[5] J. Zhang and J. G. Andrews, "Adaptive spatial intercell interference cancellation in multicell wireless networks," IEEE Journal on Selected Areas in Communications, vol. 28, no. 9, pp. 14551468, 2010.

[6] J. Zhu and H.-C. Yang, "Low-complexity coordinated beamforming transmission for multiuser MISO systems and its performance analysis," in Proceedings of the 53rd IEEE Global Communications Conference (GLOBECOM '10), Miami, Fla, USA, December 2010.

[7] U. Jang, H. Son, J. Park, and S. Lee, "CoMP-CSB for ICI nulling with user selection," IEEE Transactions on Wireless Communications, vol. 10, no. 9, pp. 2982-2993, 2011.

[8] S.-H. Moon, C. Lee, S.-R. Lee, and I. Lee, "A joint adaptive beamforming and user scheduling algorithm for downlink network MIMO systems," in Proceedings of the IEEE International Conference on Communications (ICC '13), pp. 5392-5397, Budapest, Hungary, June 2013.

[9] J. Kim, I. S. Hwang, and C. G. Kang, "Inter-cell coordinated beamforming with opportunistic scheduling," in Proceedings of the 2013 IEEE International Conference on Communications (ICC '13), pp. 5699-5703, Budapest, Hungary, June 2013.

[10] L. Zheng and D. N. C. Tse, "Diversity and multiplexing: a fundamental tradeoff in multiple-antenna channels," IEEE Transactions on Information Theory, vol. 49, no. 5, pp. 1073-1096, 2003.

[11] N. R. Goodman, "Statistical analysis based on a certain multivariate complex Gaussian distribution. (an introduction)," Annals of Mathematical Statistics, vol. 34, pp. 152-177, 1963.

[12] E. V. Haynsworth, "On the Schur complement," in Basel Mathematical Notes, University of Basel, June 1968.

[13] R. J. Muirhead, Aspects of Multivariate Statistical Theory, John Wiley \& Sons, New York, NY, USA, 1982.

[14] S. Sesia, I. Toufik, and M. Baker, LTE-The UMTS Long Term Evolution: Form Theory to Practice, Wiley, 2009.

[15] T. Muir, The Theory of Determinants in the Historical Order of Development, Dover Publications, New York, NY, USA, 1960.

[16] D. Tse and P. Viswanath, Fundamentals of Wireless Communication, Cambridge University Press, Cambridge, UK, 2005.

[17] G. Song and Y. Li, "Asymptotic throughput analysis for channelaware scheduling," IEEE Transactions on Communications, vol. 54, no. 10, pp. 1827-1834, 2006. 


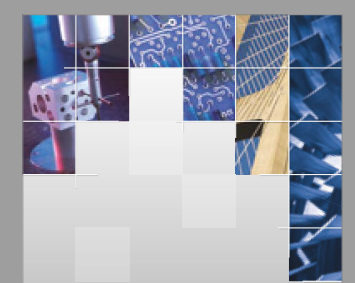

\section{Enfincering}
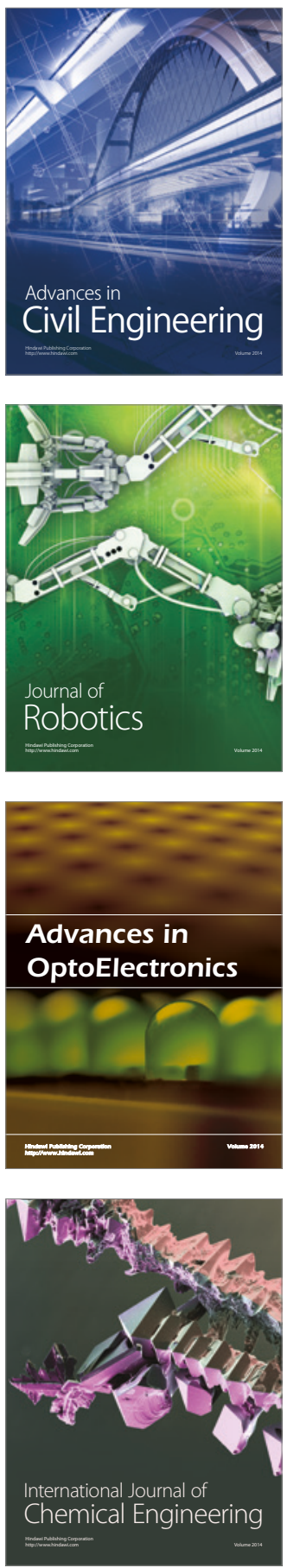

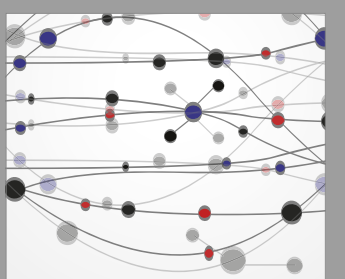

The Scientific World Journal

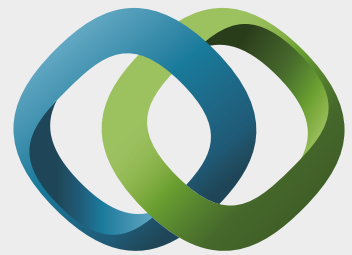

\section{Hindawi}

Submit your manuscripts at

https://www.hindawi.com
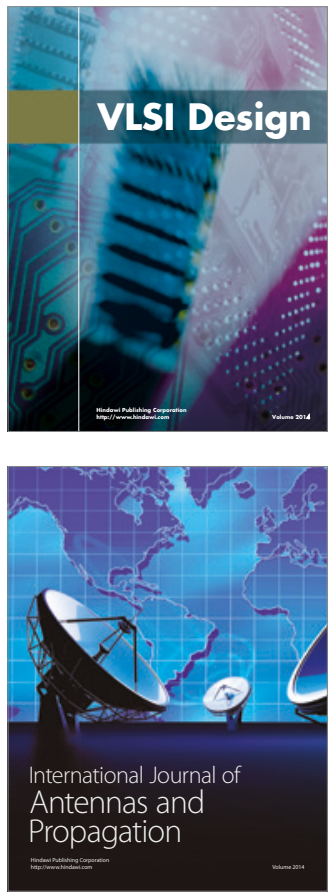

\section{Rotating}

Machinery
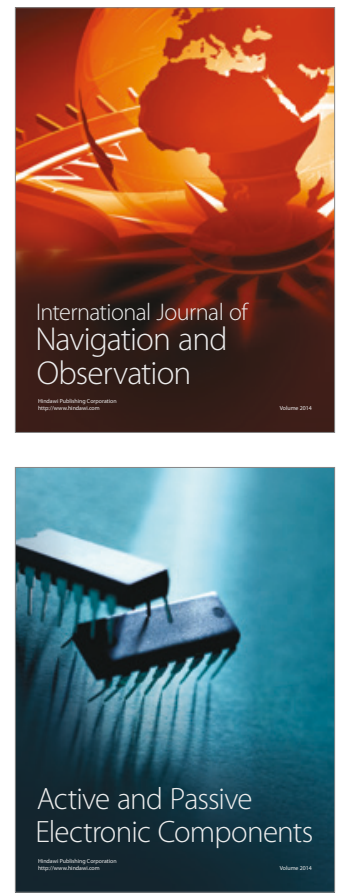
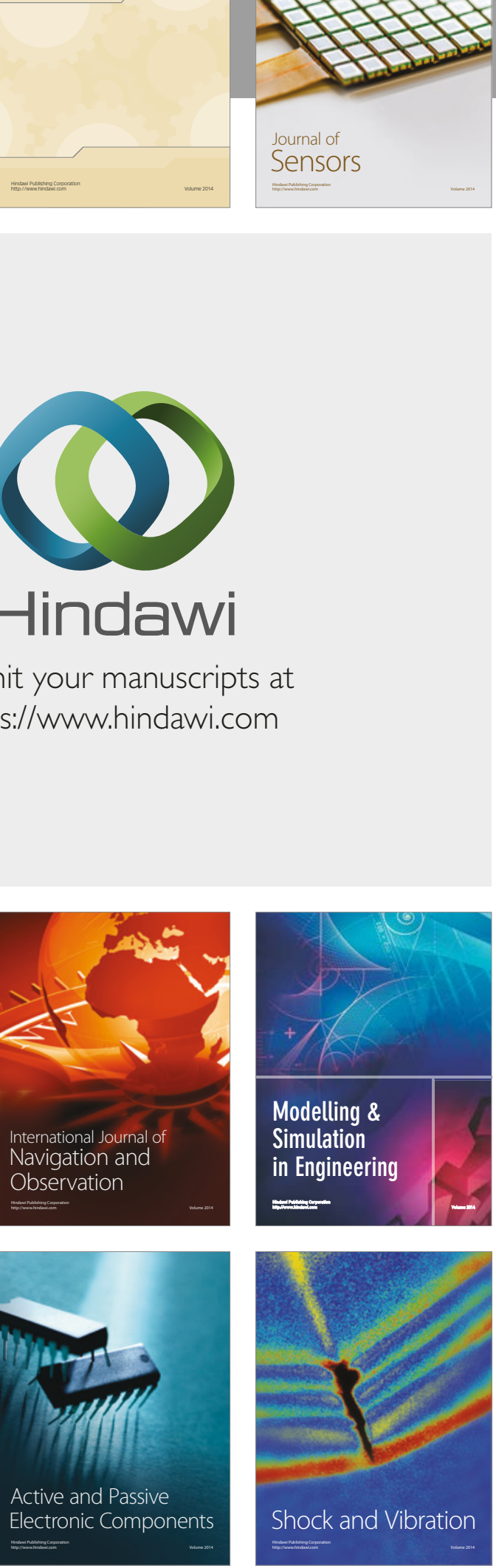
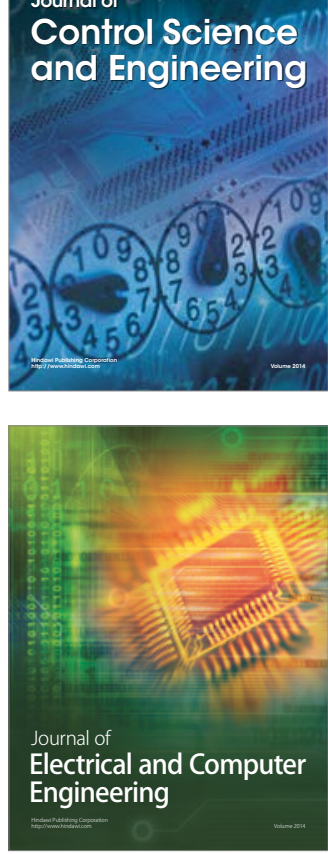

Distributed

Journal of

Control Science

and Engineering
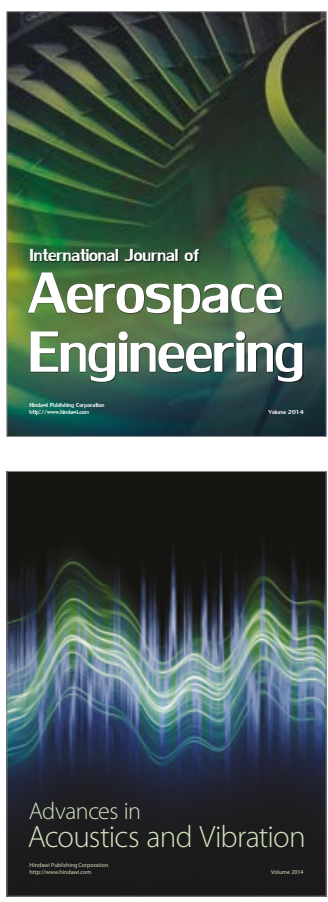

Sensor Networks 